\title{
Review of Noninvasive or Minimally Invasive Deep Brain Stimulation
}

\author{
Xiaodong Liu ${ }^{1}$, Fang $\mathrm{Qiu}^{2}$, Lijuan $\mathrm{Hou}^{3 *}$ and Xiaohui Wang ${ }^{1 *}$ \\ 'School of Kinesiology, Shanghai University of Sport, Shanghai, China, ${ }^{2}$ Department of Exercise Physiology, Beijing Sport \\ University, Beijing, China, ${ }^{3}$ College of Physical Education and Sports, Beijing Normal University, Beijing, China
}

\section{OPEN ACCESS}

Edited by:

Masaki Sekino,

The University of Tokyo, Japan

Reviewed by:

Jong-Cheol Rah,

Korea Brain Research Institute,

South Korea

Shan Huang,

University of California, Los Angeles,

United States

*Correspondence:

Lijuan Hou

houlj@bnu.edu.cn

Xiaohui Wang

wangpan96@126.com

Specialty section:

This article was submitted to

Pathological Conditions,

a section of the journal

Frontiers in Behavioral Neuroscience

Received: 22 November 2021 Accepted: 27 December 2021 Published: 18 January 2022

Citation:

Liu X, Qiu F, Hou L and Wang X

(2022) Review of Noninvasive or Minimally Invasive Deep Brain Stimulation.

Front. Behav. Neurosci. 15:820017. doi: 10.3389/fnbeh.2021.820017
Brain stimulation is a critical technique in neuroscience research and clinical application. Traditional transcranial brain stimulation techniques, such as transcranial magnetic stimulation (TMS), transcranial direct current stimulation (tDCS), and deep brain stimulation (DBS) have been widely investigated in neuroscience for decades. However, TMS and tDCS have poor spatial resolution and penetration depth, and DBS requires electrode implantation in deep brain structures. These disadvantages have limited the clinical applications of these techniques. Owing to developments in science and technology, substantial advances in noninvasive and precise deep stimulation have been achieved by neuromodulation studies. Second-generation brain stimulation techniques that mainly rely on acoustic, electronic, optical, and magnetic signals, such as focused ultrasound, temporal interference, near-infrared optogenetic, and nanomaterial-enabled magnetic stimulation, offer great prospects for neuromodulation. This review summarized the mechanisms, development, applications, and strengths of these techniques and the prospects and challenges in their development. We believe that these second-generation brain stimulation techniques pave the way for brain disorder therapy.

Keywords: deep brain stimulation, focused ultrasound, temporal interference, nanoparticle, neuromodulation

\section{INTRODUCTION}

Neuromodulation has attracted considerable attention worldwide for its value in treating neurodegenerative diseases and increasing human performance, and many countries have increased investment and built their brain projects to accelerate the development process of neuromodulation. Brain stimulation, a part of the brain project, plays a crucial role in neuroscience research and clinical application and has an advantage over pharmacotherapy because of its fast, direct, and focal effects. Brain stimulation can alter neuronal activities through the delivery of a stimulus to targeted brain areas, thus alleviating brain disorders or enhancing brain functions. Brain stimulation is a multidisciplinary research field, which involves neurophysiology, bioengineering, and material and computer science (Tatti et al., 2016; Antal et al., 2017).

The most commonly employed transcranial brain stimulation techniques include transcranial magnetic stimulation (TMS), transcranial direct current stimulation (tDCS), and deep brain stimulation (DBS; Adair et al., 2020). TMS and tDCS represent major noninvasive neurostimulation techniques, which have been widely used in clinical research for decades (Begemann et al., 2020). However, the effects of noninvasive brain stimulation through TMS and tDCS on neurons vary and are difficult to assess. Furthermore, magnetic and electric signals show absorption and scattering properties within brain tissues, limiting spatial resolution and penetration depth (Woods et al., 2016; Airan, 2017). 
DBS is an invasive neuromodulation that requires the implantation of stimulating electrodes to deep brain structures; these electrodes can precisely target deep brain nuclei through the direct control of brain circuit dynamics (Parker et al., 2020). DBS has been widely used in alleviating neurological disorders, such as motor and cognitive dysfunctions, which cannot be alleviated by traditional therapies (Lozano and Lipsman, 2013). In particular, DBS of the subthalamic nucleus (STN) is one of the most effective treatments for Parkinson's disease (Habets et al., 2018). However, DBS requires chronic implantation deep in the brain, which may eventually suffer from bleeding and infection (Kim et al., 2016). The above techniques lack cell specificity and thus have limited efficacy (Dayan et al., 2013). Thus, noninvasive and precisely deep stimulation represents a major breakthrough in neuroscience.

For this problem, exploring novel brain modulation techniques that satisfy the requirements of research and clinical application should be explored. Ideal brain stimulation targeting deep brain regions should be noninvasive or minimally invasive and have a high spatiotemporal resolution and negligible inflammatory or complications in different animal models, including rodents, large mammals, non-human primates, and humans ( $\mathrm{Li}$ et al., 2021). Great advances have been achieved by neuromodulation studies in the past decade, driven by improvements in methods and devices. In particular, second-generation brain stimulation techniques that mainly rely on acoustic, electronic, optical, and magnetic signals exhibit great promise for neuromodulation (Lewis, 2016; Lozano, 2017; Chen, 2019; Darrow, 2019). These novel techniques are aimed at surpassing the limitations of conventional brain stimulation approaches. Some current approaches are limited to laboratory research. Nevertheless, some methods have already been used in clinical applications. Here, we provide an overview of these techniques and outline the prospects and challenges in future development.

\section{FOCUSED ULTRASOUND}

Focused ultrasound (FUS) is a noninvasive neuromodulation technique with high spatial resolution and penetration depth (Figure 1; Fini and Tyler, 2017). FUS can deliver mechanical forces, penetrate biological tissues in small deep brain regions, and form a focal spot that can result in thermal and mechanical bioeffects (Kubanek, 2018; Blackmore et al., 2019). Ultrasound is a mechanical pressure wave with frequencies above the human audible range. As a propagating wave, ultrasound can alter neuronal activity by stimulating nerves and muscles (Harvey, 1929). Fry et al. (1958) first reported that ultrasound considerably affects brain activity, and they used high-intensity focused ultrasound (HIFU) for movement disorders and chronic pain (Fry, 1958). After decades of development in basic FUS technology, FUS especially Low-intensity ultrasound (LIFU) has burst a great breakthrough in scientific research and clinical treatment, continuously creating new possibilities in neuroscience (Rabut et al., 2020).

\section{Mechanisms of FUS}

FUS has many interaction modes for tissues, and these modes depend on FUS parameters, including thermal, cavitation, and mechanical mechanisms. Ultrasound can be defined as high intensity $\left(>1 \mathrm{~W} / \mathrm{cm}^{2}\right)$ or low intensity $\left(<300 \mathrm{~mW} / \mathrm{cm}^{2}\right.$; Tufail et al., 2010). The biological effects of HIFU are mainly local heating effects. The heating effects homogenize tissues and denatured proteins (Ishibashi et al., 2010). LIFU has been reported to have a great number of effects on neuromodulation. LIFU can create minimal temperature elevation. Even a small variation in temperature affects ion channels and enzymatic and potential activities (Darrow, 2019). Most studies indicated that the neuromodulation of LIFU is due to nonthermal mechanical mechanisms. Mechanosensitive ion channels, which can respond to mechanical stimuli, may mediate neural response to FUS (Ye et al., 2018). Mechanical forces exerted by FUS induce membrane displacement and mediate conformational change in embedded ion channels (Kubanek, 2018). In addition, cavitation elicited by LIFU is considered a mechanism of neuromodulation. Cavitation produces microbubbles that cause the collapse of soft tissues, and shear stress temporarily alters tight junctions and increases the permeability of the blood-brain barrier (BBB; Chu et al., 2015; Kubanek, 2018).

\section{Development and Applications of FUS}

FUS is valuable to neuroscience research and clinical applications. A large number of studies used FUS in different models, including neural tissues, rodents, non-human primates, and humans. In in vitro studies, FUS was first applied to brain modulation in the 1950s. It caused reversible suppression for sensory-evoked potential in the primary visual cortices of cats through the lateral geniculate nucleus (Fry et al., 1958). Mihran et al. (1996) determined whether or not the mechanical vibration of FUS applied to neural and cardiac cells can modify cellular excitability. Low-energy FUS increased conduction velocity and compound action potential in the excised sciatic nerves of a bullfrog (Tsui et al., 2005). In microelectrode arrays for observing the spatiotemporal dynamics of extracellular neuronal activities after FUS, local field potential spread across hippocampal sections (Suarez-Castellanos et al., 2021).

Following the initial discovery using FUS in vitro, animal behavioral effects and network activity changes have been investigated in vivo (King et al., 2013; Yu et al., 2016; Yuan et al., 2020). Tufail et al. $(2010,2011)$ reported that LIFU can promote sharp-wave ripple oscillations and trigger electromyogram (EMG) activities and forepaw and tail movements. Yuan et al. (2020) found that LIFU induces rapid hemodynamic responses at stimulation sites and demonstrated linear coupling relationships among cortical blood flow, local field potential, and EMG amplitude. Baek et al. (2018) revealed that LIFU generates motorevoked potential (MEP) and enhances sensorimotor recovery in stoke mice and found that cerebellar LIFU leads to a symmetrical decrease in pathological neural activities and enhances recovery in stoke mice. Yoo et al. (2011) demonstrated that FUS can be applied to rabbit deep brain structures and neuronal activities can be activated or suppressed depending on FUS parameters. FUS effects in large animals were further investigated, and the 


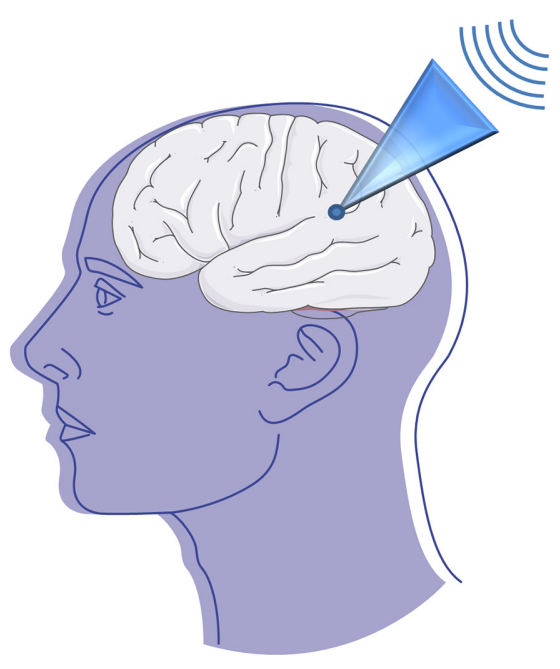

Heat

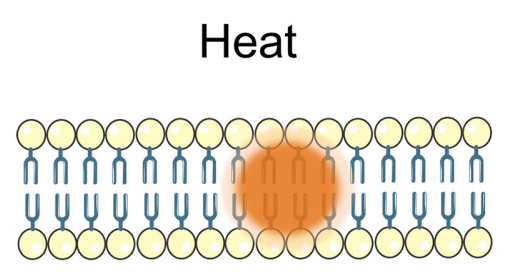

\section{Focused ultrasound}

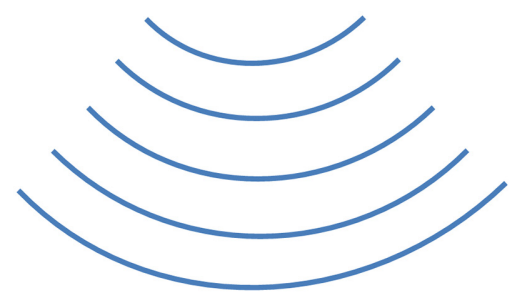

Cavitation

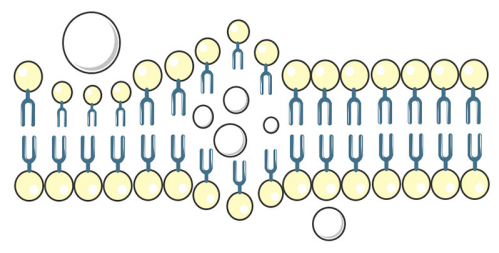

Mechanical force

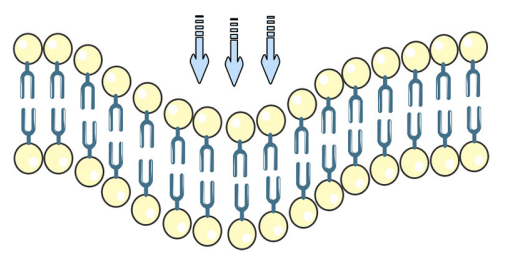

FIGURE 1 | Focused ultrasound neuromodulation. The potential biophysical effects of ultrasonic neuromodulation.

results suggested that FUS-mediated brain stimulation can be precise, effective, and safe in ovine models (Yoon et al., 2019). Deffieux et al. (2013) examined awake macaque rhesus monkeys; they showed that LIFU significantly modulates antisaccade task latencies; and they demonstrated the feasibility of using LIFU in modulating high-level cognitive behavior. What is more, FUS transiently and reversibly changes brain activities in deep cortical and subcortical regions with high spatial resolution, and modulatory effects on active and resting neurons vary (Folloni et al., 2019; Yang et al., 2021).

Moreover, the molecular responses of FUS have been recently reported. Data has shown that LIFU can stimulate brain activities involved in the activation of voltage-gated sodium and calcium channels (Tyler et al., 2008). LIFU modulates the level of neurotransmitters, Min observed a significant increase of the extracellular levels of dopamine and serotonin (Min et al., 2011). Oh et al. (2019) found that ultrasound-induced neuromodulation is initiated by transient receptor potential A1 (TRPA1) in astrocytes; TRPA1 causes a release of glutamate; finally activates $\mathrm{N}$-methyl-D-aspartic acid receptors in neighboring neurons. The expression levels of neurotrophic factors, such as brain-derived neurotrophic factor (BDNF), glial cell line-derived neurotrophic factor, and vascular endothelial growth factor, can be increased by LIFU in the rat models of Alzheimer's disease (Lin et al., 2015). BDNF expression is upregulated through the activation of tropomyosin-related kinase $\mathrm{B}$, phosphatidylinositol-3-kinase (PI3K), protein kinase B (Akt), and calmodulin kinase signaling pathways (Liu et al., 2017). FUS exposure suppresses epileptic activities in acute epilepsy rat models, and this effect seems to be mediated by the PI3K-Akt-mammalian target of rapamycin (mTOR) signaling pathway (Chen S.-G. et al., 2020).

In addition to the animal results, many researchers targeted human studies. FUS on the motor cortex transiently increases motor cortex excitability and decreases reaction time during visual motor tasks (Gibson et al., 2018; Fomenko et al., 2020). Legon et al. (2018) successfully combined electroencephalographic, computed tomography (CT), and magnetic resonance imaging (MRI) to assess the effects of FUS neuromodulation on humans. The study revealed that FUS shows perfect spatial precision and resolution when used in modulating human subcortical deep brain regions, such as the unilateral thalamus (Legon et al., 2018).

\section{Strengths and Challenges of FUS}

FUS is a promising noninvasive deep brain neuromodulation approach with high spatial precision, resolution, and safety and can reversibly modulate brain activities in subcortical and deep cortical regions with millimetric range neurostimulation (Tufail et al., 2010; Deffieux et al., 2013). Portable, wearable, and array transducer FUS has been used in research, so as to better perform its function ( $\mathrm{Li}$ et al., 2018, 2019). FUS devices are constantly developed to be more suitable for clinical practice. Using nanoparticles that specifically target drugs in specific brain areas have been used as mediators to improve the targetability 
of FUS (Ozdas et al., 2020; Hou et al., 2021). FUS has been used effectively and safely for neuromodulation in small animals, non-human primates, and humans (Legon et al., 2018; Folloni et al., 2019; Baek et al., 2020) and is compatible with MRI and CT imaging techniques, showing considerable potential as a neuromodulation method for disabling neurological disorders. Clinical trials using FUS have been conducted for the treatment of Alzheimer's disease, Parkinson's disease, epilepsy, and stroke (Meng et al., 2017; Fomenko et al., 2020).

Although a number of studies have shown that FUS is safe and effective, further prospective work is needed to elucidate parameters for safety and effectivity threshold. The shortand long-term effects of FUS need to be treated differently. Basic experiments should focus on illuminating the potential cellular, molecular, synaptic, and ionic mechanisms of FUS neuromodulation.

\section{TEMPORAL INTERFERENCE STIMULATION}

Temporal interference (TI) stimulation is a novel noninvasive transcranial electrical stimulation (TES) technique that can reach deep brain regions (Figure 2). It utilizes multigroup high frequency $(\geq 1,000 \mathrm{~Hz})$ and oscillating electric fields in modulating neural activities. In 1965, The TI concept was proposed by Russian scientists and applied to peripheral stimulation (Beatti et al., 2011; Guleyupoglu et al., 2013; Li et al., 2020). Then, Grossman et al. (2017) used TI stimulation in brain research in 2017. Since then, TI has attracted the attention of neuroscientists because it may achieve noninvasive deep brain stimulation. The position of TI stimulation can be changed by adjusting electrode location and current amplitude ratio. It can target deep brain regions and prevent the activation of the neighboring and overlying cortex. TI stimulation offers a means to precisely regulate subcortical structures.

\section{Mechanisms of TI Stimulation}

TI stimulation consists of two sets of high frequency sinusoidal waveform currents with slightly different frequencies $\left(f_{1}=2,000 \mathrm{~Hz} ; f_{2}=2,010 \mathrm{~Hz} ; \Delta f=10 \mathrm{~Hz}\right)$. Two high frequency oscillating electric fields interact and produce an envelope that is equal to $\Delta \mathrm{f}$ in current intersection regions. Owing to neural biophysical properties, neural membranes respond to low-frequency electrical signals, and high-frequency oscillation does not recruit effective neural firing (Hutcheon and Yarom, 2000). Cao et al. (2018) demonstrated that neurons exhibit TI stimulation in a single neuron computational model. Esmaeilpour et al. (2021) investigated that the spatial selectivity of TI stimulation in deep brain areas depends on the phasic modulation of neural oscillations. TI stimulation can modulate spiking activity and facilitate phase synchronization, similar to transcranial alternating current stimulation (tACS; Howell and McIntyre, 2021). At similar field intensities, TI stimulation has less potent modulatory effects than other conventional TES (Negahbani et al., 2018). Mirzakhalili et al. (2020) found that TI stimulation requires a signal rectification process mediated by ion channels. The subthreshold neuromodulation of TI

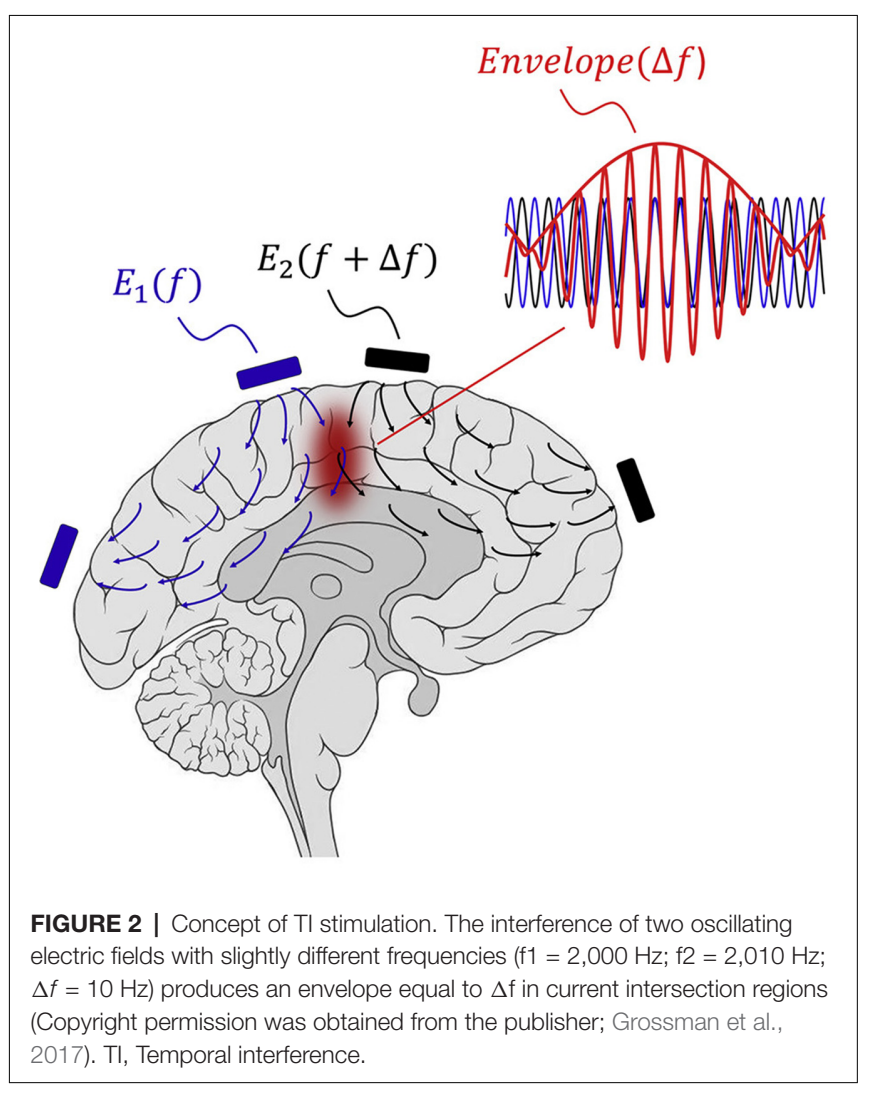

stimulation may be the most important effect (Chakraborty et al., 2018), and polarization effects can alter neural firing and synaptic transmission. Moreover, the potential mechanisms of TI stimulation may involve neurons, glial cells, and cerebral blood flow (Wachter et al., 2011; Monai et al., 2016). More studies are needed to explore and clarify the possible mechanisms.

\section{Development and Applications of $\mathrm{TI}$ Stimulation}

Grossman et al. (2017) proposed that TI stimulation is noninvasive deep brain stimulation and carried out a series of experiments to validate the approach (Bouthour et al., 2017). Mouse neurons were fired with the $\Delta \mathrm{f}$ envelopes of electric fields with a patch clamp electrophysiological recording technique. To assess the focality and depth of TI stimulation, they applied $10 \mathrm{~Hz}$ of transcranial stimulation and $2,000 \mathrm{~Hz}+2,010 \mathrm{~Hz}$ TI stimulation to the hippocampi of anesthetized mice and then measured the expression of the immediate early gene $c$ fos (an indicator of activated neurons). Transcranial stimulation at $10 \mathrm{~Hz}$ results in widespread expression in the cortex and hippocampus. By contrast, 2,000 Hz $+2,010 \mathrm{~Hz}$ of TI stimulation activates hippocampus regions without activating the cortex. They also explored behavioral responses to TI stimulation and found that TI stimulation can evoke forepaw movement. Stimulation regions can be altered by changing the ratios of currents without electrode movement. No pathophysiological activities and neural damage were observed (Grossman et al., 2017). Using the finite element method, Lee et al. (2020) 
found that optimized TI stimulation can successfully reach the hippocampus while reducing the effect of neocortical regions. Another study designed a TI stimulation method and validated its steerability through finite element analysis by using an action potential model and measuring waveforms in a saline solution (Xiao et al., 2019). The simulations of TI stimulation in a mouse head model achieved field strength in deep brain areas but less field strength in superficial areas (Grossman et al., 2017). Furthermore, the field strengths in a human model were much lower, and no direct stimulation effects were found; current higher than that in a mouse model might be required (Rampersad et al., 2019). Computational results indicated that the activation threshold increased with frequency and the envelope frequency had no association with the threshold. Moreover, the current intensity ratio altered the position of responding neurons. The characteristics of an envelope may predict the regions of TI stimulation (Gomez-Tames et al., 2021). Multichannel array electrodes for TI stimulation enhance focality and reduce scalp sensation in computational modeling and mouse experiments (Song et al., 2020). Wang H. et al. (2020) fabricated a TI stimulator that measures bioimpedance in real time and proposed an approach that can easily locate the target position. Current investigations on TI stimulation mainly use computational simulations and small animal experiments. A study investigated the variability in the electric field during TI stimulation and compared it to tACS. The results showed that the electric fields of TI stimulation are variable and more focal than those of tACS (von Conta et al., 2021). Hence, experiments on human subjects are necessary. Further human investigations on TI stimulation needs to be validated.

\section{Strengths and Challenges of TI Stimulation}

In summary, the prospects of TI stimulation using noninvasive techniques are exciting. Conventional noninvasive TES usually generates scalp pain when exposing stimulation and limits the intensity of injection currents (Wu et al., 2021). TI stimulation can selectively stimulate specific brain regions, such as cortical and subcortical areas, thus preventing the stimulation of scalp nerves and scalp pain (Gomez-Tames et al., 2021). Given that DBS has remarkable therapeutic benefits for the treatment of Parkinson's disease, tremor, and dystonia (Kringelbach et al., 2007), TI stimulation as a noninvasive DBS offers exciting prospects for the treatment of various brain disorders.

Most TI stimulation studies focus on computation and animal models, and thus human trials need to be further investigated. Given that anatomical differences affect electric field distributions, optimal TI stimulation parameters need to be further validated using various models. Furthermore, a specific positioning scheme for target regions is currently unavailable. An optimization algorithm focusing on the electric field in a target region should be established. Electrode fixation and interference location calculation should be accurate and convenient to facilitate clinical translation (Gunduz and Okun, 2017). TI stimulation currently cannot match the spatial resolution of implantable DBS. Deep small brain structures may not be specifically stimulated, such as the subthalamic nucleus (Grossman, 2018). Further studies are necessary to elucidate the related mechanism, which may involve neurons, synaptic plasticity, cerebral blood flow, and glial cells (Mirzakhalili et al., 2020). More importantly, the safety of TI stimulation needs to be examined and monitored. Finally, the prospect of TI stimulation neuromodulation method is highly promising, but the method requires further research before it can be applied to clinical processes.

\section{NEAR-INFRARED OPTOGENETIC STIMULATION}

Near-infrared (NIR) optogenetic stimulation is a mode of optogenetic stimulation that does not require optical fiber implantation and has minimal invasiveness. Optogenetics is widely used in exploring neural circuits that govern sensory, memory, and motor behavior (Hausser, 2014). However, optogenetics requires the insertion of invasive optical fibers to target areas because the penetration depth of visible light is shallow. Blue-green wavelength penetration is limited because of the scattering and absorption by endogenous chromophores (Lin et al., 2013). NIR light (650-1,350 nm), which is much less scattered, can easily penetrate tissues and reach deep brain areas (Shi et al., 2016). NIR light-based photoregulation strategies offer means to modulate specific cells in deep brain structures (Chen G. et al., 2020). However, NIR light cannot be used directly and requires special processing. NIR optogenetic approaches stimulate deep brain regions by activating channel rhodopsin-expressing neurons, where NIR light is converted to visible light (Figure 3). By using this approach, researchers can control behavioral patterns simply by NIR illumination without performing optical fiber implantation (Chen et al., 2018). This approach shows great potential in bioimaging and neuromodulation because of its low imaging background and deep penetration (Wu et al., 2015).

\section{Mechanisms of NIR Optogenetic Stimulation}

NIR optogenetic stimulation needs NIR light nanotransducers to exert its function. Typically, lanthanide-doped upconversion nanoparticles (UCNPs) can be modulated to a particular wavelength because of the special ladder-like electronic energy structures of trivalent lanthanide ions (Zhou et al., 2015). Dopant-host combination can control the emission wavelengths of UCNPs (Wang and Liu, 2009). The different site symmetries of dopant ions affect emission wavelength and emission peak intensity. The output color of UCNPs can be adjusted into a specific wavelength for optogenetic stimulation (All et al., 2019). UCNPs can convert low-energy NIR light to high-energy visible light (Prodi et al., 2015). UCNPs can be implanted close to optogenetic opsin neurons, and NIR illumination can be converted into visible light, which in turn activates optogenetic opsins, regulates light-gated ion channels that control the, and outflow of ions, and induces cell activation or suppression (Yu et al., 2019). Moreover, blended UCNPs with distinct excitation and emission wavelengths may result in neuron excitation and inhibition simultaneously within one region or multiple deep brain areas (Chen, 2019). 


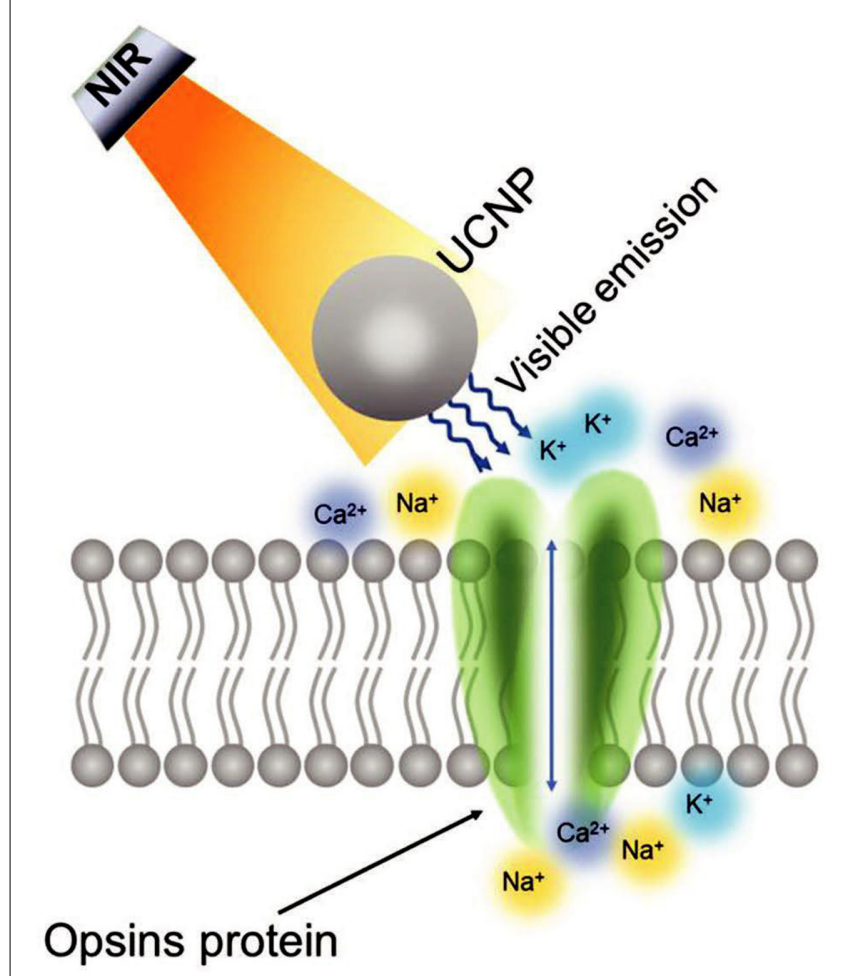

FIGURE 3 | Near-infrared optogenetic stimulation. Schematic principle of lanthanide-doped upconversion nanoparticle (UCNP) mediated NIR optogenetic stimulation (Copyright permission was obtained from the publisher; Yu et al., 2019).

\section{Development and Applications of NIR Optogenetic Stimulation}

UCNP-mediated optogenetics was first proposed by Deisseroth in 2011 (Chen S. et al., 2020). After a decade of research and development, NIR optogenetic stimulation has been investigated using different models (Ai et al., 2017; Wang et al., 2017; Ding et al., 2018). Hososhima et al. (2015) first used cultured cells containing UCNPs to observe the photoreactive responses that express channelrhodopsin. Neurons are triggered by NIR laser irradiation and generate action potential (Hososhima et al., 2015). Wu et al. (2016) synergized two unconversion booster effectors (dye-sensitizing and core/shell enhancement) to enhance upconversion efficiency; they successfully altered optogenetic neuron excitation to a biocompatible, watersolubilized, and deep-tissue penetrable $800 \mathrm{~nm}$ wavelength. The first in vivo study was investigated with Caenorhabditis elegans (C. elegans). C. elegans is widely used in optogenetic manipulation because of its small nervous system and quantifiable motor behavior (Zhen and Samuel, 2015). Bansal et al. (2016) implemented NIR optogenetic stimulation in $C$. elegans and found that it can activate channelrhodopsin-2 ion channels in mechanosensory neurons at a low average power with a quasi-continuous wave excitation approach. Further study showed that UCNPs can effectively activate inhibitory GABAergic motor neurons and excitatory glutamatergic DVC interneurons, leading to locomotion inhibition and activation (Ao et al., 2019). A recent study on zebrafish showed that NIR optogenetic stimulation can remotely activate channelrhodopsin-2 ion channels and effectively manipulate cation influx. This investigation provided a site-specific approach for regulating membrane-associated activities (Ai et al., 2017). More importantly, NIR optogenetic stimulation on live rodent animals was conducted in a number of experimental studies. Lin et al. (2017) packaged UCNPs in glass micropipettes and positioned them close to specific brain structures, such as the tegmental area, cortical striatum, and visual cortex. The results showed that NIR light remotely activated targeting brain structures and showed great biocompatibility (Lin et al., 2017). They then implanted a microscale upconversionbased device into a mouse brain and successfully controlled motor function in awake and freely moving animal (Wang et al., 2017; Lin et al., 2018). NIR optogenetic stimulation represented a major leap when Chen et al. published their findings in the Science journal (Feliu et al., 2018). They demonstrated that UCNP-mediated NIR approach regulated multiple neuronal activities in deep brain regions, specifically evoking dopamine release in the ventral tegmental area, inducing brain oscillations by activating the medial septum, and silencing seizure by inhibiting hippocampal cells and triggering memory recall. In addition, the study showed the excellent biocompatibility, flexibility, robust minimal invasiveness, long-term in vivo utility, low dispersion, and negligible cytotoxicity of the approach (Chen et al., 2018). One large timescale study demonstrated that NIR optogenetic stimulation successfully controlled animal locomotive behavior by manipulating neurons in the dorsal striatum and UCNPs remained functional for at least 8 weeks at the injection brain site; these results suggested that using this approach in long-term behavioral tests is highly feasible (Miyazaki et al., 2019). Ma et al. (2019) reported that injected UCNPs enable retinal photoreceptors to perceive NIR light and differentiate sophisticated NIR shape patterns. This type of vision is compatible with daylight vision, offering options for mammalian vision repair and enhancement (Ma et al., 2019). Liu et al. (2021) developed NIR multicolor optogenetics using trichromatic UCNPs, which can selectively activate three distinct neuronal populations and modulate motor behavior in awake mice.

\section{Strengths and Challenges of NIR Optogenetic Stimulation}

NIR optogenetic stimulation offers the possibility of delivering light to deep brain regions, is less invasiveness, and has a high spatial resolution and cell specificity (Chen S. et al., 2020). Compared with optogenetics, NIR optogenetic stimulation can be manipulated remotely in the brain without resulting in behavior restriction (All et al., 2019; Lin et al., 2021). This approach has been validated in vitro and in vivo in terms of its capability to modulate neural activities, and the results suggested potential neuroscience applications. Safety has been demonstrated in many studies, as well as good biocompatibility and negligible toxicity. Furthermore, advancements in NIR optogenetic stimulation require collaboration among physicists, 
chemists, neuroscientists, and biologists. It is a big step toward the remote and noninvasive control of brain activities. Hence, transferring this approach to clinical trials is possible, and it may complement current neurological disorder therapies, such as DBS.

Some challenges encountered in NIR optogenetic stimulation need to be addressed here. The toxicity of UCNPs on the cellular, tissue, and organ levels should be comprehensively investigated in order that potential organ damage can be prevented, and long-term biocompatibility studies should be conducted given that UCNPs may change properties and are readily endocytosed by cells (Nazarenus et al., 2014). Effective nanostructures should be designed to satisfy different studies (Tao et al., 2020). In addition, further investigations using large animals are required before clinical trials. Despite such challenges, the recent discovery of NIR optogenetic stimulation is a significant breakthrough. We believe that this new technology has bright therapeutic prospects.

\section{NANOMATERIAL-ENABLED MAGNETIC STIMULATION}

Magnetic fields can penetrate tissues with less attenuation and harmless effects, thus having potential uses in wireless and noninvasive methods for modulating brain activities (Christiansen et al., 2019). Magnetic fields are considered intermediary and should be converted to localized secondary stimuli (Wang and Guo, 2016). Methods combining magnetic fields with magnetic nanoparticles (MNPs) converting magnetic signals have been investigated with different techniques (Huang et al., 2010; Wang G. et al., 2020; Kozielski et al., 2021). MNPs as transducers can be categorized into magnetothermal activation, magnetoelectric activation, and magnetomechanical activation (Roet et al., 2019). MNPs incorporate ion-transporting proteins, which can be transgenically expressed in neurons and respond to changes in heat, electricity, or force (Christiansen et al., 2019). It is commonly known as nanomaterial-enabled magnetic stimulation. This approach represents a more effective stimulation that can noninvasively modulate deep brain neural activities and selectively activate specific neural circuits.

\section{Mechanisms of Nanomaterial-Enabled Magnetic Stimulation}

Magnetothermal activation uses alternating magnetic fields (AMFs) to activate the temperature-gated ion channels of transient receptor potential vanilloid (TRPV) family (Figure 4). MNPs can fuse to TRPV and generate heat through hysteretic power loss and then induce calcium ion influx, membrane depolarization, and action potential firing (Huang et al., 2010; Munshi et al., 2017). TRPV1 is endogenously expressed in mammalian neurons (Starowicz et al., 2008; Terzian et al., 2014). Some studies used genetic tools to achieve the uniform expression of TRPV1 in specific brain areas in mice (Huang et al., 2010; Temel and Jahanshahi, 2015). Magnetoelectric activation uses magnetoelectric nanoparticles (MENs) to generate local electric fields under an external magnetic field. The electric field originates from the intrinsic coupling between electric and magnetic fields in MENs (Guduru et al., 2015). Magnetomechanical activation uses MNPs to convert the energy of magnetic fields into mechanical forces (Chen M. et al., 2020). These forces can activate pressure-sensitive receptors and subsequently modulate neurons (Shin and Cheon, 2017).

\section{Development and Applications of Nanomaterial-Enabled Magnetic Stimulation}

Huang et al. (2010) first demonstrated that superparamagnetic nanoparticles exposed to AMFs can locally generate heat and remotely activate TRPV1, eliciting responses from human embryonic kidney 293 cells and C. elegans. Another study showed that modified TRPV1 with MNPs can modulate calcium influx in vivo and in vitro when exposed to a magnetic field (Stanley et al., 2012). Further studies aimed to determine whether a magnetic field can regulate the behavior of rodents animals. Radio wave or magnetic field treatment for glucokinase-Cre (GK-Cre) mice that received ventromedial hypothalamus injection of Ad-FLEX-anti-GFP-TRPV1/GFPferritin alters blood glucose and food intake (Stanley et al., 2016). However, the above investigations did not discuss the mechanisms of neural modulation. Chen et al. (2015) exerted a considerable amount of effort into studying wireless magnetothermal activation. In mice, the hysteretic heating of MNPs activates hippocampal and ventral tegmental area neurons after the application of AMFs. To ensure the sustained and uniform levels of TRPV1 expression, the author designed a transgene across a cell membrane. Meanwhile, magnetothermal deep brain stimulation has minimal cytotoxicity, long-term, biocompatibility, and stability (Chen et al., 2015). Munshi et al. (2017) first reported magnetothermal activation using MNPs in awake and freely moving animals. Magnetothermal stimulation in the motor cortex or striatum evokes different types of motor behavior, and the duration of behavior correlates with magnetic field application (Munshi et al., 2017). In addition, they transfected rat hippocampal neurons to express thermosensitive chloride channel anoctamin 1 and silenced neuronal activity by applying a magnetic field to target neurons (Munshi et al., 2018). The behavioral responses evoked by magnetothermal activation result from optogenetic or chemogenetic neural modulation. Hescham et al. (2021) used a wireless magnetothermal approach for parkinsonian-like mice. The results revealed that magnetothermal neuromodulation in the STN can not only modulate the motor behavior of healthy mice remotely but also reverse motor deficits (Hescham et al., 2021). MNPs offer attractive methods for brain tumor therapies because magnetic fields can stimulate tumors through heating without damaging healthy hypodermal tissues (Thorat et al., 2016, 2019). This approach prevents the serious adverse effects of traditional chemotherapy. Compared with magnetothermal activation, magnetoelectric activation, and magnetomechanical activation have not been extensively explored. Nguyen et al. (2021) intravenously injected MENs 

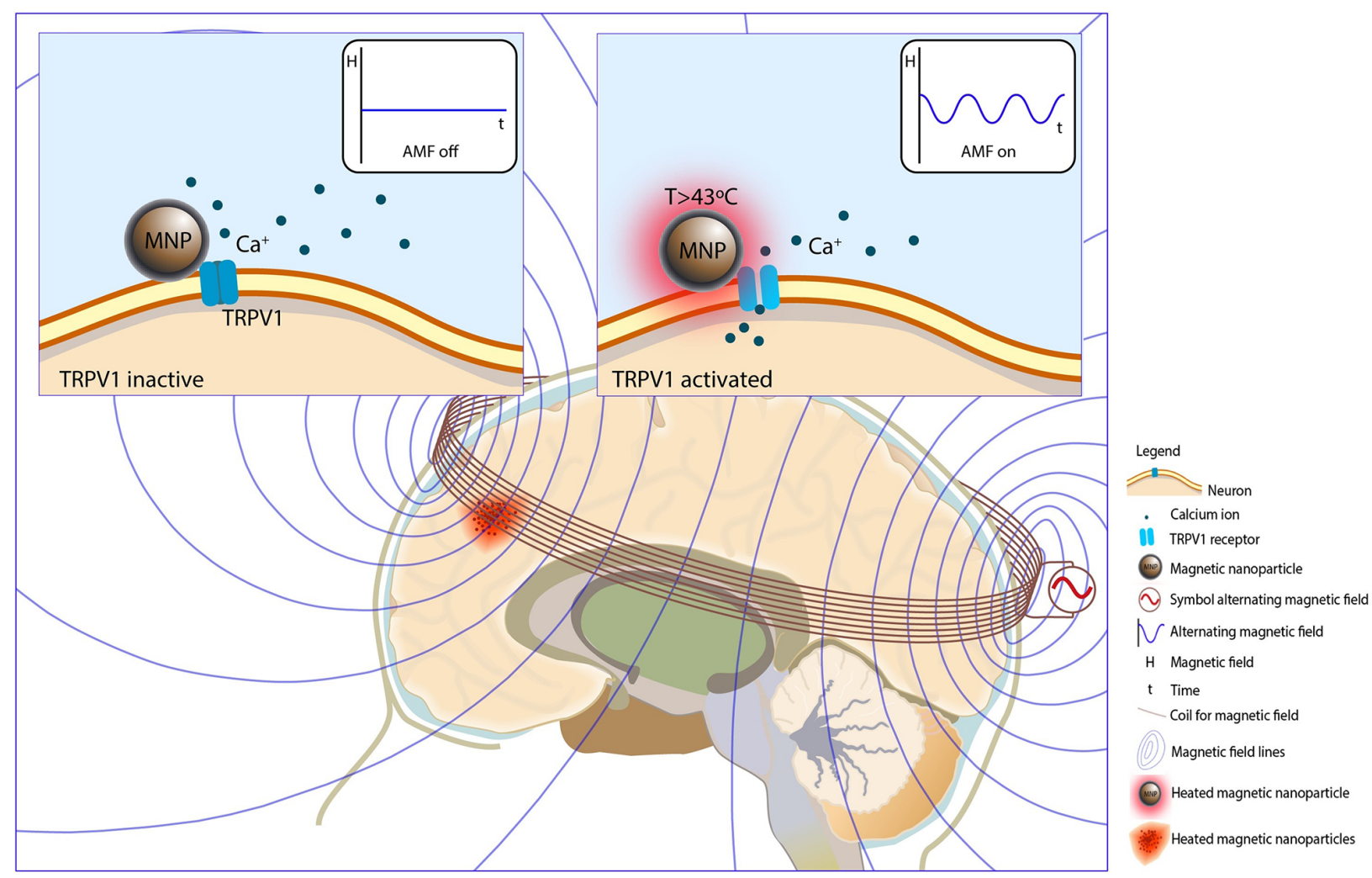

Roet, M. Progress in Neurobiology

FIGURE 4 | Nanomaterial-enabled magnetic stimulation. A schematic view and schematic principle of nanomaterial-enabled magnetic stimulation in the human brain (Copyright permission was obtained from the publisher; Roet et al., 2019).

TABLE 1 | Overview of each type of neuromodulation.

\begin{tabular}{|c|c|c|c|c|}
\hline & FUS & TI stimulation & $\begin{array}{l}\text { NIR optogenetic } \\
\text { stimulation }\end{array}$ & $\begin{array}{l}\text { Nanomaterial-enabled magnetic } \\
\text { stimulation }\end{array}$ \\
\hline Energy delivery & Ultrasound & Electrical & Near-infrared & Magnetic \\
\hline Invasiveness & Noninvasive & Noninvasive & Minimally invasive & Minimally invasive \\
\hline Spatial resolution & $\sim 1 \mathrm{~mm}$ & $>\mathrm{mm}$ & $<1 \mathrm{~mm}$ & $<1 \mathrm{~mm}$ \\
\hline Depth of penetration & $10-15 \mathrm{~cm}$ or more & $5 \mathrm{~cm}$ or more & $1 \mathrm{~cm}$ or more & Unlimited in theory \\
\hline Gene delivery & No & No & Yes & Yes \\
\hline Experiment animal models & $\begin{array}{l}\text { Rodents, non-human primates, } \\
\text { human }\end{array}$ & Rodents, human & Rodents & Rodents \\
\hline Stimulation mode & Fixing transducer & Fixing electrodes & Remote & Remote \\
\hline Complexity level & Moderate & Moderate & Complicated & Complicated \\
\hline Reversible & Yes & Yes & No & No \\
\hline Cost & Moderate & Low & High & High \\
\hline
\end{tabular}

FUS, focused ultrasound.

and forced them to cross the $\mathrm{BBB}$ and localize to the cortical areas by using a magnetic field gradient. The results showed that cortical neurons and cortical networks can be activated by an external magnetic field (Nguyen et al., 2021). Kozielski et al. (2021) demonstrated that the magnetic stimulation of MENs can modulate neuronal activities in the motor cortex and nonmotor thalamus and modulate mice behavior. Overall, nanomaterial-enabled magnetic stimulation may facilitate remote noninvasive deep brain stimulation without genetic manipulation.

\section{Strengths and Challenges of Nanomaterial-Enabled Magnetic Stimulation}

Nanomaterial-enabled magnetic stimulation has offered broad application prospects for noninvasive deep brain modulation. The approach provides high spatial resolution and cell specificity. Its feasibility, effectiveness, biocompatibility, stability, and safety have been validated in vitro and in vivo (Chen et al., 2015; Park et al., 2020; Wang G. et al., 2020; Kozielski et al., 2021). More importantly, nanomaterial-enabled magnetic stimulation 
can utilize MNPs for the modulation of neurons with heat or electric or mechanical forces without genetically engineering. This feature is important and may ensure clinical trial approval (Starowicz et al., 2008). The chemical composition of MNPs is similar to that of MRI agents by having minimal cytotoxicity and long-term effectiveness (Petters et al., 2014; Roet et al., 2019). Meanwhile, with the development of nanotechnology, MNPs have huge biomedical application potential (Chen et al., 2017; Manescu Paltanea et al., 2021).

Studies on nanomaterial-enabled magnetic stimulation mainly focused on small animal models. The next step should be conducting studies on non-human primates and even clinical trials. Scaling AMF coils to human deep brain areas is a huge challenge. In addition, The heating side effects of MNPs should be considered because they may result in brain swelling and increase intracranial pressure (Maier-Hauff et al., 2007). Moreover, heating can promote MNP aggregation, which may cause occlusion in the blood vessel (Wegscheid et al., 2014) and ultimately lead to serious clinical consequences. Therefore, solving this problem is highly necessary. Lastly, the long-term toxicological effects and clearance of MNPs in the brain regions should be investigated.

\section{FUTURE TRENDS}

The above deep brain neuromodulation techniques mainly rely on acoustic, electronic, optical, and magnetic signals and show great promise as a high-spatiotemporal resolution and deep penetration platform. These approaches are noninvasive or minimally invasive. The characteristics of the four types of neuromodulation are summarized in Table $\mathbf{1}$.

FUS and TI stimulation are noninvasive neuromodulations without gene delivery. It is relatively easy to translate to the clinic. These techniques may serve as complementary neuromodulation for the treatment of brain disorders. We believe that FUS and TI could be an upgrade of traditional DBS to improve efficiency and safety. However, a similar situation as DBS, FUS, and TI stimulation may just alleviate the progression but cannot cure the disease. Furthermore, none of these approaches has cell type-specific to the brain target. Therefore, future research should explore the underlying mechanisms behind FUS and TI

\section{REFERENCES}

Adair, D., Truong, D., Esmaeilpour, Z., Gebodh, N., Borges, H., Ho, L., et al. (2020). Electrical stimulation of cranial nerves in cognition and disease. Brain Stimul. 13, 717-750. doi: 10.1016/j.brs.2020.02.019

Ai, X., Lyu, L., Zhang, Y., Tang, Y., Mu, J., Liu, F., et al. (2017). Remote regulation of membrane channel activity by site-specific localization of lanthanidedoped upconversion nanocrystals. Angew. Chem. Int. Ed. Engl. 56, 3031-3035. doi: 10.1002/anie.201612142

Airan, R. (2017). Neuromodulation with nanoparticles. Science 357:465. doi: $10.1126 /$ science.aao 1200

All, A. H., Zeng, X., Teh, D. B. L., Yi, Z., Prasad, A., Ishizuka, T., et al. (2019). Expanding the toolbox of upconversion nanoparticles for in vivo optogenetics and neuromodulation. Adv. Mater 31:e1803474. doi: 10.1002/adma.201803474 stimulation so that the results can be optimized for clinical application.

Compared to FUS and TI stimulation, NIR optogenetic stimulation and nanomaterial-enabled magnetic stimulation have a long way to go for clinical application. Both approaches need gene delivery. So there are a number of practical challenges before clinical application. First, the long-term safety of viral vectors used for genetic modification to the target neurons has yet to be fully illustrated. Second, maintaining the gene delivery effective and stable in different animal models especially non-human primates is also a potential challenge. Third, genetic therapy for primates is much more complicated, and high cost and long cycle are required for the research. Therefore, there remains much to be done before NIR optogenetic stimulation and nanomaterial-enabled magnetic stimulation can be delivered to the clinical arena. Promisingly, gene therapy has been increasingly applied to treat tumors, virus infection, and genetic disease. As gene delivery technologies develop, the application will be continuously updated. That would be of great significance in neuromodulation.

\section{CONCLUSIONS}

These techniques may represent next-generation neural interface tools for neuroscience and have huge potential as tools for advancing neuroscience research. Cross-disciplinary collaboration is needed to establish an optimal scheme given and confirm that these techniques are indeed next-generation noninvasive DBS technologies. We believe that advancements in these techniques will pave the way for novel therapeutic options for brain disorders.

\section{AUTHOR CONTRIBUTIONS}

XL and FQ wrote the manuscript. LH performed revision and improved the quality of the manuscript. XW edited and revised the manuscript. All authors contributed to the article and approved the submitted version.

\section{FUNDING}

This work was supported by a grant from the key program of National Natural Science Foundation of China (No. 11932013).

Antal, A., Alekseichuk, I., Bikson, M., Brockmöller, J., Brunoni, A. R., Chen, R., et al. (2017). Low intensity transcranial electric stimulation: Safety, ethical, legal regulatory and application guidelines. Clin. Neurophysiol. 128, 1774-1809. doi: 10.1016/j.clinph.2017.06.001

Ao, Y., Zeng, K., Yu, B., Miao, Y., Hung, W., Yu, Z., et al. (2019). An upconversion nanoparticle enables near infrared-optogenetic manipulation of the caenorhabditis elegans motor circuit. ACS Nano 13, 3373-3386. doi: 10.1021/acsnano.8b09270

Baek, H., Pahk, K. J., Kim, M. J., Youn, I., and Kim, H. (2018). Modulation of cerebellar cortical plasticity using low-intensity focused ultrasound for poststroke sensorimotor function recovery. Neurorehabil. Neural Repair 32, 777-787. doi: 10.1177/1545968318790022

Baek, H., Sariev, A., Lee, S., Dong, S. Y., Royer, S., and Kim, H. (2020) Deep cerebellar low-intensity focused ultrasound stimulation restores 
interhemispheric balance after ischemic stroke in mice. IEEE Trans. Neural Syst. Rehabil. Eng. 28, 2073-2079. doi: 10.1109/TNSRE.2020.30 02207

Bansal, A., Liu, H., Jayakumar, M. K., Andersson-Engels, S., and Zhang, Y. (2016). Quasi-continuous wave near-infrared excitation of upconversion nanoparticles for optogenetic manipulation of C. elegans. Small 12, 1732-1743. doi: 10.1002/smll.201503792

Beatti, A., Rayner, A., Chipchase, L., and Souvlis, T. (2011). Penetration and spread of interferential current in cutaneous, subcutaneous and muscle tissues. Physiotherapy 97, 319-326. doi: 10.1016/j.physio.2011.01.008

Begemann, M. J., Brand, B. A., Curcic-Blake, B., Aleman, A., and Sommer, I. E. (2020). Efficacy of non-invasive brain stimulation on cognitive functioning in brain disorders: a meta-analysis. Psychol. Med. 50, 2465-2486. doi: $10.1017 / S 0033291720003670$

Blackmore, J., Shrivastava, S., Sallet, J., Butler, C. R., and Cleveland, R. O. (2019). Ultrasound neuromodulation: a review of results, mechanisms and safety. Ultrasound Med. Biol. 45, 1509-1536. doi: 10.1016/j.ultrasmedbio.2018.12.015

Bouthour, W., Krack, P., and Luscher, C. (2017). A deeply superficial brain stimulation. Mov. Disord. 32:1326. doi: 10.1002/mds.27111

Cao, J., and Grover, P. (2018). "Do single neuron models exhibit temporal interference stimulation," in 2018 IEEE Biomedical Circuits and Systems Conference (BioCAS). doi: 10.1109/BIOCAS.2018.8584745

Chakraborty, D., Truong, D. Q., Bikson, M., and Kaphzan, H. (2018). Neuromodulation of axon terminals. Cereb. Cortex 28, 2786-2794. doi: 10.1093/cercor/bhx158

Chen, S. (2019). Optical modulation goes deep in the brain. Science 365, 456-457. doi: $10.1126 /$ science.aay4350

Chen, G., Cao, Y., Tang, Y., Yang, X., Liu, Y., Huang, D., et al. (2020). Advanced near-infrared light for monitoring and modulating the spatiotemporal dynamics of cell functions in living systems. Adv. Sci. (Weinh) 7:1903783. doi: 10.1002/advs.201903783

Chen, R., Canales, A., and Anikeeva, P. (2017). Neural recording and modulation technologies. Nat. Rev. Mater 2:16093. doi: 10.1038/natrevmats.2016.93

Chen, R., Romero, G., Christiansen, M. G., Mohr, A., and Anikeeva, P. (2015). Wireless magnetothermal deep brain stimulation. Science 347, 1477-1480. doi: $10.1126 /$ science. 1261821

Chen, S.-G., Tsai, C.-H., Lin, C.-J., Lee, C.-C., Yu, H.-Y., Hsieh, T.-H., et al. (2020). Transcranial focused ultrasound pulsation suppresses pentylenetetrazol induced epilepsy in vivo. Brain Stimul. 13, 35-46. doi: 10.1016/j.brs.2019. 09.011

Chen, S., Weitemier, A. Z., Zeng, X., He, L., Wang, X., Tao, Y., et al. (2018). Near-infrared deep brain stimulation via upconversion nanoparticle-mediated optogenetics. Science 359, 679-684. doi: 10.1126/science.aaq1144

Chen, S., Wu, J., Cai, A., Gonzalez, N., and Yin, R. (2020). Towards minimally invasive deep brain stimulation and imaging: a near-infrared upconversion approach. Neurosci. Res. 152, 59-65. doi: 10.1016/j.neures.2020.01.005

Chen, M., Wu, J., Ning, P., Wang, J., Ma, Z., Huang, L., et al. (2020). Remote control of mechanical forces via mitochondrial-targeted magnetic nanospinners for efficient cancer treatment. Small 16:e1905424. doi: 10.1002/smll.201905424

Christiansen, M. G., Senko, A. W., and Anikeeva, P. (2019). Magnetic strategies for nervous system control. Annu. Rev. Neurosci. 42, 271-293. doi: 10.1146/annurev-neuro-070918-050241

Chu, P. C., Liu, H. L., Lai, H. Y., Lin, C. Y., Tsai, H. C., and Pei, Y. C. (2015). Neuromodulation accompanying focused ultrasound-induced bloodbrain barrier opening. Sci. Rep. 5:15477. doi: 10.1038/srep15477

Darrow, D. P. (2019). Focused ultrasound for neuromodulation. Neurotherapeutics 16, 88-99. doi: 10.1007/s13311-018-00691-3

Dayan, E., Censor, N., Buch, E. R., Sandrini, M., and Cohen, L. G. (2013). Noninvasive brain stimulation: from physiology to network dynamics and back. Nat. Neurosci. 16, 838-844. doi: 10.1038/nn.3422

Deffieux, T., Younan, Y., Wattiez, N., Tanter, M., Pouget, P., and Aubry, J. F. (2013). Low-intensity focused ultrasound modulates monkey visuomotor behavior. Curr. Biol. 23, 2430-2433. doi: 10.1016/j.cub.2013.10.029

Ding, H., Lu, L., Shi, Z., Wang, D., Li, L., Li, X., et al. (2018). Microscale optoelectronic infrared-to-visible upconversion devices and their use as injectable light sources. Proc. Natl. Acad. Sci. U S A 115, 6632-6637. doi: 10.1073/pnas. 1802064115
Esmaeilpour, Z., Kronberg, G., Reato, D., Parra, L. C., and Bikson, M. (2021). Temporal interference stimulation targets deep brain regions by modulating neural oscillations. Brain Stimul. 14, 55-65. doi: 10.1016/j.brs.2020. 11.007

Feliu, N., Neher, E., and Parak, W. J. (2018). Toward an optically controlled brain. Science 359, 633-634. doi: 10.1126/science.aar7379

Fini, M., and Tyler, W. J. (2017). Transcranial focused ultrasound: a new tool for non-invasive neuromodulation. Int. Rev. Psychiatry 29, 168-177. doi: 10.1080/09540261.2017.1302924

Folloni, D., Verhagen, L., Mars, R. B., Fouragnan, E., Constans, C., Aubry, J.F., et al. (2019). Manipulation of subcortical and deep cortical activity in the primate brain using transcranial focused ultrasound stimulation. Neuron 101, 1109-1116.e5. doi: 10.1016/j.neuron.2019.01.019

Fomenko, A., Chen, K. S., Nankoo, J. F., Saravanamuttu, J., Wang, Y., ElBaba, M., et al. (2020). Systematic examination of low-intensity ultrasound parameters on human motor cortex excitability and behavior. eLife 9:e54497. doi: $10.7554 /$ eLife.54497

Fry, F., Ades, H., and Fry, W. J. S. (1958). Production of reversible changes in the central nervous system by ultrasound. Science $127,83-84$. doi: 10.1126/science. 127.3289 .83

Fry, W. J. (1958). Use of intense ultrasound in neurological research. Am. J. Phys. Med. 37, 143-147.

Gibson, B. C., Sanguinetti, J. L., Badran, B. W., Yu, A. B., Klein, E. P., Abbott, C. C., et al. (2018). Increased excitability induced in the primary motor cortex by transcranial ultrasound stimulation. Front. Neurol. 9:1007. doi: 10.3389/fneur. 2018.01007

Gomez-Tames, J., Asai, A., and Hirata, A. (2021). Multiscale computational model reveals nerve response in a mouse model for temporal interference brain stimulation. Front. Neurosci. 15:684465. doi: 10.3389/fnins.2021.684465

Grossman, N. (2018). Modulation without surgical intervention. Science 361, 461-462. doi: 10.1126/science.aau4915

Grossman, N., Bono, D., Dedic, N., Kodandaramaiah, S. B., Rudenko, A., Suk, H. J., et al. (2017). Noninvasive deep brain stimulation via temporally interfering electric fields. Cell 169, 1029-1041.e16. doi: 10.1016/j.cell.2017.05.024

Guduru, R., Liang, P., Hong, J., Rodzinski, A., Hadjikhani, A., Horstmyer, J., et al. (2015). Magnetoelectric "spin" on stimulating the brain. Nanomedicine (Lond) 10, 2051-2061. doi: 10.2217/nnm.15.52

Guleyupoglu, B., Schestatsky, P., Edwards, D., Fregni, F., and Bikson, M. (2013). Classification of methods in transcranial electrical stimulation (tES) and evolving strategy from historical approaches to contemporary innovations. J. Neurosci. Methods 219, 297-311. doi: 10.1016/j.jneumeth.2013.07.016

Gunduz, A., and Okun, M. S. (2017). A new non-surgical approach for deep-brain stimulation. Lancet Neurol. 16:e1. doi: 10.1016/S1474-4422(17)30224-7

Habets, J. G. V., Heijmans, M., Kuijf, M. L., Janssen, M. L. F., Temel, Y., and Kubben, P. L. (2018). An update on adaptive deep brain stimulation in Parkinson's disease. Mov. Disord. 33, 1834-1843. doi: 10.1002/mds.115

Harvey, E. N. (1929). The effect of high frequency sound waves on heart muscle and other irritable tissues. Am. J. Physiol. 91, 284-290. doi: 10.1152/ajplegacy. 1929.91.1.284

Hausser, M. (2014). Optogenetics: the age of light. Nat. Methods 11, 1012-1014. doi: $10.1038 /$ nmeth.3111

Hescham, S. A., Chiang, P. H., Gregurec, D., Moon, J., Christiansen, M. G., Jahanshahi, A., et al. (2021). Magnetothermal nanoparticle technology alleviates parkinsonian-like symptoms in mice. Nat. Commun. 12:5569. doi: 10.1038/s41467-021-25837-4

Hososhima, S., Yuasa, H., Ishizuka, T., Hoque, M. R., Yamashita, T., Yamanaka, A., et al. (2015). Near-infrared (NIR) up-conversion optogenetics. Sci. Rep. 5:16533. doi: 10.1038/srep16533

Hou, X., Qiu, Z., Xian, Q., Kala, S., Jing, J., Wong, K. F., et al. (2021). Precise ultrasound neuromodulation in a deep brain region using nano gas vesicles as actuators. Adv. Sci. (Weinh) 8:e2101934. doi: 10.1002/advs.202101934

Howell, B., and McIntyre, C. C. (2021). Feasibility of interferential and pulsed transcranial electrical stimulation for neuromodulation at the human scale. Neuromodulation 24, 843-853. doi: 10.1111/ner.13137

Huang, H., Delikanli, S., Zeng, H., Ferkey, D. M., and Pralle, A. (2010). Remote control of ion channels and neurons through magnetic-field heating of nanoparticles. Nat. Nanotechnol. 5, 602-606. doi: 10.1038/nnano. 2010.125 
Hutcheon, B., and Yarom, Y. (2000). Resonance, oscillation and the intrinsic frequency preferences of neurons. Trends Neurosci. 23, 216-222. doi: 10.1016/s0166-2236(00)01547-2

Ishibashi, K., Shimada, K., Kawato, T., Kaji, S., Maeno, M., Sato, S., et al. (2010). Inhibitory effects of low-energy pulsed ultrasonic stimulation on cell surface protein antigen $\mathrm{C}$ through heat shock proteins GroEL and DnaK in Streptococcus mutans. Appl. Environ. Microbiol. 76, 751-756. doi: 10.1128/AEM.02230-09

Kim, M.-R., Yun, J. Y., Jeon, B., Lim, Y. H., Kim, K. R., Yang, H.-J., et al. (2016). Patients' reluctance to undergo deep brain stimulation for Parkinson's disease. Parkinsonism Relat. Disord. 23, 91-94. doi: 10.1016/j.parkreldis.2015. 11.010

King, R. L., Brown, J. R., Newsome, W. T., and Pauly, K. B. (2013). Effective parameters for ultrasound-induced in vivo neurostimulation. Ultrasound Med. Biol. 39, 312-331. doi: 10.1016/j.ultrasmedbio.2012.09.009

Kozielski, K. L., Jahanshahi, A., Gilbert, H. B., Yu, Y., Erin, Ö., Francisco, D., et al. (2021). Nonresonant powering of injectable nanoelectrodes enables wireless deep brain stimulation in freely moving mice. Sci. Adv. 7:eabc4189. doi: 10.1126/sciadv.abc4189

Kringelbach, M. L., Jenkinson, N., Owen, S. L. F., and Aziz, T. Z. (2007). Translational principles of deep brain stimulation. Nat. Rev. Neurosci. 8, 623-635. doi: 10.1038/nrn2196

Kubanek, J. (2018). Neuromodulation with transcranial focused ultrasound. Neurosurg. Focus 44:E14. doi: 10.3171/2017.11.FOCUS17621

Lee, S., Lee, C., Park, J., and Im, C. H. (2020). Individually customized transcranial temporal interference stimulation for focused modulation of deep brain structures: a simulation study with different head models. Sci. Rep. 10:11730. doi: 10.1038/s41598-020-68660-5

Legon, W., Ai, L., Bansal, P., and Mueller, J. K. (2018). Neuromodulation with single-element transcranial focused ultrasound in human thalamus. Hum. Brain Mapp. 39, 1995-2006. doi: 10.1002/hbm.23981

Lewis, S. (2016). Techniques: magnetic manipulation. Nat. Rev. Neurosci. 17, 262-263. doi: 10.1038/nrn.2016.42

Li, J., Lee, K. M., and Bai, K. (2020). Analytical and experimental investigation of temporal interference for selective neuromuscular activation. IEEE Trans. Neural Syst. Rehabil. Eng. 28, 3100-3112. doi: 10.1109/TNSRE.2020.30 38025

Li, G., Qiu, W., Hong, J., Jiang, Q., Su, M., Mu, P., et al. (2018). Imaging-guided dual-target neuromodulation of the mouse brain using array ultrasound. IEEE Trans. Ultrason. Ferroelectr. Freq. Control 65, 1583-1589. doi: 10.1109/TUFFC. 2018.2847252

Li, G., Qiu, W., Zhang, Z., Jiang, Q., Su, M., Cai, R., et al. (2019). Noninvasive ultrasonic neuromodulation in freely moving mice. IEEE Trans. Biomed. Eng. 66, 217-224. doi: 10.1109/TBME.2018.2821201

Li, X., Xiong, H., Rommelfanger, N., Xu, X., Youn, J., Slesinger, P. A., et al. (2021). Nanotransducers for wireless neuromodulation. Matter 4, 1484-1510. doi: 10.1016/j.matt.2021.02.012

Lin, W. T., Chen, R. C., Lu, W. W., Liu, S. H., and Yang, F. Y. (2015). Protective effects of low-intensity pulsed ultrasound on aluminum-induced cerebral damage in Alzheimer's disease rat model. Sci. Rep. 5:9671. doi: 10.1038/srep09671

Lin, X., Chen, X., Zhang, W., Sun, T., Fang, P., Liao, Q., et al. (2018). Coreshell-shell upconversion nanoparticles with enhanced emission for wireless optogenetic inhibition. Nano. Lett. 18, 948-956. doi: 10.1021/acs.nanolett. $7 \mathrm{~b} 04339$

Lin, J. Y., Knutsen, P. M., Muller, A., Kleinfeld, D., and Tsien, R. Y. (2013). ReaChR: a red-shifted variant of channelrhodopsin enables deep transcranial optogenetic excitation. Nat. Neurosci. 16, 1499-1508. doi: 10.1038/nn.3502

Lin, X., Wang, Y., Chen, X., Yang, R., Wang, Z., Feng, J., et al. (2017). Multiplexed optogenetic stimulation of neurons with spectrum-selective upconversion nanoparticles. Adv. Healthc. Mater. 6:1700446. doi: 10.1002/adhm. 201700446

Lin, Y., Yao, Y., Zhang, W., Fang, Q., Zhang, L., Zhang, Y., et al. (2021). Applications of upconversion nanoparticles in cellular optogenetics. Acta Biomater. 135, 1-12. doi: 10.1016/j.actbio.2021.08.035

Liu, X., Chen, H., Wang, Y., Si, Y., Zhang, H., Li, X., et al. (2021). Near-infrared manipulation of multiple neuronal populations via trichromatic upconversion. Nat. Commun. 12:5662. doi: 10.1038/s41467-021-25993-7
Liu, S. H., Lai, Y. L., Chen, B. L., and Yang, F. Y. (2017). Ultrasound enhances the expression of brain-derived neurotrophic factor in astrocyte through activation of TrkB-Akt and Calcium-CaMK signaling pathways. Cereb. Cortex 27, 3152-3160. doi: 10.1093/cercor/bhw169

Lozano, A. M. (2017). Waving hello to noninvasive deep-brain stimulation. N Engl. J. Med. 377, 1096-1098. doi: 10.1056/NEJMcibr1707165

Lozano, A. M., and Lipsman, N. (2013). Probing and regulating dysfunctional circuits using deep brain stimulation. Neuron 77, 406-424. doi: 10.1016/j. neuron.2013.01.020

Ma, Y., Bao, J., Zhang, Y., Li, Z., Zhou, X., Wan, C., et al. (2019). Mammalian near-infrared image vision through injectable and self-powered retinal nanoantennae. Cell 177, 243-255.e15. doi: 10.1016/j.cell.2019. 01.038

Maier-Hauff, K., Rothe, R., Scholz, R., Gneveckow, U., Wust, P., Thiesen, B., et al. (2007). Intracranial thermotherapy using magnetic nanoparticles combined with external beam radiotherapy: results of a feasibility study on patients with glioblastoma multiforme. J. Neurooncol. 81, 53-60. doi: 10.1007/s11060-0069195-0

Manescu Paltanea, V., Paltanea, G., Antoniac, I., and Vasilescu, M. (2021). Magnetic nanoparticles used in oncology. Materials (Basel, Switzerland) 14:5948. doi: 10.3390/ma14205948

Meng, Y., Volpini, M., Black, S., Lozano, A. M., Hynynen, K., and Lipsman, N. (2017). Focused ultrasound as a novel strategy for Alzheimer disease therapeutics. Ann. Neurol. 81, 611-617. doi: 10.1002/ana.24933

Mihran, R. T., Lineaweaver, S. K., Barnes, F. S., and Wachtel, H. (1996). Effects of pulsed acoustic and mechanical stimuli on the excitability of isolated neuronal and cardiac cells. Appl. Occup. Env. Hygiene 11, 271-274. doi: 10.1080/1047322X.1996.10389322

Min, B.-K., Yang, P. S., Bohlke, M., Park, S., R.Vago, D., Maher, T. J., et al. (2011). Focused ultrasound modulates the level of cortical neurotransmitters: potential as a new functional brain mapping technique. Int. J. Imaging Syst. Technol. 21, 232-240. doi: 10.1002/ima.20284

Mirzakhalili, E., Barra, B., Capogrosso, M., and Lempka, S. F. (2020). Biophysics of temporal interference stimulation. Cell Syst. 11, 557-572.e5. doi: 10.1016/j.cels. 2020.10.004

Miyazaki, T., Chowdhury, S., Yamashita, T., Matsubara, T., Yawo, H., Yuasa, H., et al. (2019). Large timescale interrogation of neuronal function by fiberless optogenetics using lanthanide micro-particles. Cell Rep. 26, 1033-1043.e5. doi: 10.1016/j.celrep.2019.01.001

Monai, H., Ohkura, M., Tanaka, M., Oe, Y., Konno, A., Hirai, H., et al. (2016). Calcium imaging reveals glial involvement in transcranial direct current stimulation-induced plasticity in mouse brain. Nat. Commun. 7:11100. doi: $10.1038 /$ ncomms 11100

Munshi, R., Qadri, S. M., and Pralle, A. (2018). Transient magnetothermal neuronal silencing using the chloride channel anoctamin 1 (TMEM16A). Front. Neurosci. 12:560. doi: 10.3389/fnins.2018.00560

Munshi, R., Qadri, S. M., Zhang, Q., Castellanos Rubio, I., Del Pino, P., and Pralle, A. (2017). Magnetothermal genetic deep brain stimulation of motor behaviors in awake, freely moving mice. eLife 6:e27069. doi: 10.7554/eLife. 27069

Nazarenus, M., Zhang, Q., Soliman, M. G., Del Pino, P., Pelaz, B., CarregalRomero, S., et al. (2014). in vitro interaction of colloidal nanoparticles with mammalian cells: what have we learned thus far. Beilstein J. Nanotechnol. 5, 1477-1490. doi: 10.3762/bjnano.5.161

Negahbani, E., Kasten, F. H., Herrmann, C. S., and Frohlich, F. (2018). Targeting alpha-band oscillations in a cortical model with amplitudemodulated high-frequency transcranial electric stimulation. Neuroimage 173 , 3-12. doi: 10.1016/j.neuroimage.2018.02.005

Nguyen, T., Gao, J., Wang, P., Nagesetti, A., Andrews, P., Masood, S., et al. (2021). In vivo wireless brain stimulation via non-invasive and targeted delivery of magnetoelectric nanoparticles. Neurotherapeutics 18, 2091-2106. doi: 10.1007/s13311-021-01071-0

Oh, S. J., Lee, J. M., Kim, H. B., Lee, J., Han, S., Bae, J. Y., et al. (2019). Ultrasonic neuromodulation via astrocytic TRPA1. Curr. Biol. 29, 3386-3401.e8. doi: 10.1016/j.cub.2019.08.021

Ozdas, M. S., Shah, A. S., Johnson, P. M., Patel, N., Marks, M., Yasar, T. B., et al. (2020). Non-invasive molecularly-specific millimeter-resolution manipulation of brain circuits by ultrasound-mediated aggregation and 
uncaging of drug carriers. Nat. Commun. 11:4929. doi: 10.1038/s41467-02018059-7

Park, J., Tabet, A., Moon, J., Chiang, P. H., Koehler, F., Sahasrabudhe, A., et al. (2020). Remotely controlled proton generation for neuromodulation. Nano Lett. 20, 6535-6541. doi: 10.1021/acs.nanolett.0c02281

Parker, T., Raghu, A. L. B., FitzGerald, J. J., Green, A. L., and Aziz, T. Z. (2020). Multitarget deep brain stimulation for clinically complex movement disorders. J. Neurosurg. 134, 351-356. doi: 10.3171/2019.11.JNS192224

Petters, C., Irrsack, E., Koch, M., and Dringen, R. (2014). Uptake and metabolism of iron oxide nanoparticles in brain cells. Neurochem. Res. 39, 1648-1660. doi: 10.1007/s11064-014-1380-5

Prodi, L., Rampazzo, E., Rastrelli, F., Speghini, A., and Zaccheroni, N. (2015). Imaging agents based on lanthanide doped nanoparticles. Chem. Soc. Rev. 44, 4922-4952. doi: 10.1039/c4cs00394b

Rabut, C., Yoo, S., Hurt, R. C., Jin, Z., Li, H., Guo, H., et al. (2020). Ultrasound technologies for imaging and modulating neural activity. Neuron 108, 93-110. doi: 10.1016/j.neuron.2020.09.003

Rampersad, S., Roig-Solvas, B., Yarossi, M., Kulkarni, P. P., Santarnecchi, E., Dorval, A. D., et al. (2019). Prospects for transcranial temporal interference stimulation in humans: a computational study. Neuroimage 202:116124. doi: 10.1016/j.neuroimage.2019.116124

Roet, M., Hescham, S. A., Jahanshahi, A., Rutten, B. P. F., Anikeeva, P. O., and Temel, Y. (2019). Progress in neuromodulation of the brain: a role for magnetic nanoparticles. Prog. Neurobiol. 177, 1-14. doi: 10.1016/j.pneurobio.2019.03.002

Shi, L., Sordillo, L. A., Rodriguez-Contreras, A., and Alfano, R. (2016). Transmission in near-infrared optical windows for deep brain imaging. J. Biophotonics 9, 38-43. doi: 10.1002/jbio.201500192

Shin, T.-H., and Cheon, J. (2017). Synergism of nanomaterials with physical stimuli for biology and medicine. Acc. Chem. Res. 50, 567-572. doi: 10.1021/acs. accounts.6b00559

Song, X., Zhao, X., Li, X., Liu, S., and Ming, D. (2020). Multi-channel transcranial temporally interfering stimulation (tTIS): application to living mice brain. J. Neural Eng. doi: 10.1088/1741-2552/abd2c9. [Online ahead of print].

Stanley, S. A., Gagner, J. E., Damanpour, S., Yoshida, M., Dordick, J. S., and Friedman, J. M. (2012). Radio-wave heating of iron oxide nanoparticles can regulate plasma glucose in mice. Science 336, 604-608. doi: 10.1126/science. 1216753

Stanley, S. A., Kelly, L., Latcha, K. N., Schmidt, S. F., Yu, X., Nectow, A. R., et al. (2016). Bidirectional electromagnetic control of the hypothalamus regulates feeding and metabolism. Nature 531, 647-650. doi: 10.1038/nature17183

Starowicz, K., Cristino, L., and Di Marzo, V. (2008). TRPV1 receptors in the central nervous system: potential for previously unforeseen therapeutic applications. Curr. Pharm. Design 14, 42-54. doi: 10.2174/138161208783330790

Suarez-Castellanos, I. M., Dossi, E., Vion-Bailly, J., Salette, L., Chapelon, J.Y., Carpentier, A., et al. (2021). Spatio-temporal characterization of causal electrophysiological activity stimulated by single pulse Focused Ultrasound: an ex vivo study on hippocampal brain slices. J. Neural Eng. doi: 10.1088/17412552/abdfb1. [Online ahead of print].

Tao, Y., Chan, H. F., Shi, B., Li, M., and Leong, K. W. (2020). Light: a magical tool for controlled drug delivery. Adv. Funct. Mater. 30:2005029. doi: 10.1002/adfm. 202005029

Tatti, E., Rossi, S., Innocenti, I., Rossi, A., and Santarnecchi, E. (2016). Noninvasive brain stimulation of the aging brain: State of the art and future perspectives. Ageing Res. Rev. 29, 66-89. doi: 10.1016/j.arr.2016.05.006

Temel, Y., and Jahanshahi, A. (2015). Neuroscience. treating brain disorders with neuromodulation. Science 347, 1418-1419. doi: 10.1126/science.aaa9610

Terzian, A. L., dos Reis, D. G., Guimaraes, F. S., Correa, F. M., and Resstel, L. B. (2014). Medial prefrontal cortex transient receptor potential vanilloid type 1 (TRPV1) in the expression of contextual fear conditioning in Wistar rats. Psychopharmacology (Berl) 231, 149-157. doi: 10.1007/s00213-0133211-9

Thorat, N. D., Bohara, R. A., Malgras, V., Tofail, S. A. M., Ahamad, T., Alshehri, S. M., et al. (2016). Multimodal superparamagnetic nanoparticles with unusually enhanced specific absorption rate for synergetic cancer therapeutics and magnetic resonance imaging. ACS Appl. Mater. Interfaces 8, 14656-14664. doi: $10.1021 /$ acsami.6b02616

Thorat, N. D., Townely, H., Brennan, G., Parchur, A. K., Silien, C., Bauer, J., et al. (2019). Progress in remotely triggered hybrid nanostructures for next-generation brain cancer theranostics. ACS Biomater. Sci. Eng. 5, 2669-2687. doi: 10.1021/acsbiomaterials.8b01173

Tsui, P. H., Wang, S. H., and Huang, C. C. (2005). in vitro effects of ultrasound with different energies on the conduction properties of neural tissue. Ultrasonics 43, 560-565. doi: 10.1016/j.ultras.2004.12.003

Tufail, Y., Matyushov, A., Baldwin, N., Tauchmann, M. L., Georges, J., Yoshihiro, A., et al. (2010). Transcranial pulsed ultrasound stimulates intact brain circuits. Neuron 66, 681-694. doi: 10.1016/j.neuron.2010.05.008

Tufail, Y., Yoshihiro, A., Pati, S., Li, M. M., and Tyler, W. J. (2011). Ultrasonic neuromodulation by brain stimulation with transcranial ultrasound. Nat Protoc. 6, 1453-1470. doi: 10.1038/nprot.2011.371

Tyler, W. J., Tufail, Y., Finsterwald, M., Tauchmann, M. L., Olson, E. J., and Majestic, C. (2008). Remote excitation of neuronal circuits using lowintensity, low-frequency ultrasound. PLoS One 3:e3511. doi: 10.1371/journal. pone.0003511

von Conta, J., Kasten, F. H., Curcic-Blake, B., Aleman, A., Thielscher, A., and Herrmann, C.S. (2021). Interindividual variability of electric fields during transcranial temporal interference stimulation (tTIS). Sci. Rep. 11:20357. doi: 10.1038/s41598-021-99749-0

Wachter, D., Wrede, A., Schulz-Schaeffer, W., Taghizadeh-Waghefi, A., Nitsche, M. A., Kutschenko, A., et al. (2011). Transcranial direct current stimulation induces polarity-specific changes of cortical blood perfusion in the rat. Exp. Neurol. 227, 322-327. doi: 10.1016/j.expneurol.2010.12.005

Wang, Y., and Guo, L. (2016). Nanomaterial-enabled neural stimulation. Front. Neurosci. 10:69. doi: 10.3389/fnins.2016.00069

Wang, F., and Liu, X. (2009). Recent advances in the chemistry of lanthanide-doped upconversion nanocrystals. Chem. Soc. Rev. 38, 976-989. doi: $10.1039 / \mathrm{b} 809132 \mathrm{n}$

Wang, Y., Lin, X., Chen, X., Chen, X., Xu, Z., Zhang, W., et al. (2017). Tetherless near-infrared control of brain activity in behaving animals using fully implantable upconversion microdevices. Biomaterials 142, 136-148. doi: 10.1016/j.biomaterials.2017.07.017

Wang, H., Shi, Z., Sun, W., Zhang, J., Wang, J., Shi, Y., et al. (2020). Development of a non-invasive deep brain stimulator with precise positioning and real-time monitoring of bioimpedance. Front. Neuroinform. 14:574189. doi: 10.3389/fninf.2020.574189

Wang, G., Zhang, P., Mendu, S. K., Wang, Y., Zhang, Y., Kang, X., et al. (2020). Revaluation of magnetic properties of Magneto. Nat. Neurosci. 23, 1047-1050. doi: 10.1038/s41593-019-0473-5

Wegscheid, M. L., Morshed, R. A., Cheng, Y., and Lesniak, M. S. (2014). The art of attraction: applications of multifunctional magnetic nanomaterials for malignant glioma. Exp. Opin. Drug Deliv. 11, 957-975. doi: 10.1517/17425247. 2014.912629

Woods, A. J., Antal, A., Bikson, M., Boggio, P. S., Brunoni, A. R., Celnik, P., et al. (2016). A technical guide to tDCS and related non-invasive brain stimulation tools. Clin. Neurophysiol. 127, 1031-1048. doi: 10.1016/j.clinph.2015.11.012

Wu, X., Chen, G., Shen, J., Li, Z., Zhang, Y., and Han, G. (2015). Upconversion nanoparticles: a versatile solution to multiscale biological imaging. Bioconjug. Chem. 26, 166-175. doi: 10.1021/bc5003967

Wu, L., Liu, T., and Wang, J. (2021). Improving the effect of transcranial alternating current stimulation (tACS): a systematic review. Front. Hum. Neurosci. 15:652393. doi: 10.3389/fnhum.2021.652393

Wu, X., Zhang, Y., Takle, K., Bilsel, O., Li, Z., Lee, H., et al. (2016). Dyesensitized core/active shell upconversion nanoparticles for optogenetics and bioimaging applications. ACS Nano 10, 1060-1066. doi: 10.1021/acsnano.5b 06383

Xiao, Q., Zhong, Z., Lai, X., and Qin, H. (2019). A multiple modulation synthesis method with high spatial resolution for noninvasive neurostimulation. PLoS One 14:e0218293. doi: 10.1371/journal.pone. 0218293

Yang, P. F., Phipps, M. A., Jonathan, S., Newton, A. T., Byun, N., Gore, J. C., et al. (2021). Bidirectional and state-dependent modulation of brain activity by transcranial focused ultrasound in non-human primates. Brain Stimul. 14, 261-272. doi: 10.1016/j.brs.2021.01.006

Ye, J., Tang, S., Meng, L., Li, X., Wen, X., Chen, S., et al. (2018). Ultrasonic control of neural activity through activation of the mechanosensitive channel MscL. Nano Lett. 18, 4148-4155. doi: 10.1021/acs.nanolett. $8 \mathrm{~b} 00935$ 
Yoo, S. S., Bystritsky, A., Lee, J. H., Zhang, Y., Fischer, K., Min, B. K., et al. (2011). Focused ultrasound modulates region-specific brain activity. Neuroimage 56, 1267-1275. doi: 10.1016/j.neuroimage.2011.02.058

Yoon, K., Lee, W., Lee, J. E., Xu, L., Croce, P., Foley, L., et al. (2019). Effects of sonication parameters on transcranial focused ultrasound brain stimulation in an ovine model. PLoS One 14:e0224311. doi: 10.1371/journal.pone. 0224311

Yu, N., Huang, L., Zhou, Y., Xue, T., Chen, Z., and Han, G. (2019). Nearinfrared-light activatable nanoparticles for deep-tissue-penetrating wireless optogenetics. Adv. Healthc. Mater. 8:e1801132. doi: 10.1002/adhm.201 801132

Yu, K., Sohrabpour, A., and He, B. (2016). Electrophysiological source imaging of brain networks perturbed by low-intensity transcranial focused ultrasound. IEEE Trans. Biomed. Eng. 63, 1787-1794. doi: 10.1109/TBME.2016.25 91924

Yuan, Y., Wang, Z., Liu, M., and Shoham, S. (2020). Cortical hemodynamic responses induced by low-intensity transcranial ultrasound stimulation of mouse cortex. Neuroimage 211:116597. doi: 10.1016/j.neuroimage.2020.116597

Zhen, M., and Samuel, A. D. (2015). C. elegans locomotion: small circuits, complex functions. Curr. Opin. Neurobiol. 33, 117-126. doi: 10.1016/j.conb.2015. 03.009
Zhou, B., Shi, B., Jin, D., and Liu, X. (2015). Controlling upconversion nanocrystals for emerging applications. Nat. Nanotechnol. 10, 924-936. doi: 10.1038/nnano. 2015.251

Conflict of Interest: The authors declare that the research was conducted in the absence of any commercial or financial relationships that could be construed as a potential conflict of interest.

Publisher's Note: All claims expressed in this article are solely those of the authors and do not necessarily represent those of their affiliated organizations, or those of the publisher, the editors and the reviewers. Any product that may be evaluated in this article, or claim that may be made by its manufacturer, is not guaranteed or endorsed by the publisher.

Copyright (c) $2022 \mathrm{Liu}$, Qiu, Hou and Wang. This is an open-access article distributed under the terms of the Creative Commons Attribution License (CC BY). The use, distribution or reproduction in other forums is permitted, provided the original author (s) and the copyright owner (s) are credited and that the original publication in this journal is cited, in accordance with accepted academic practice. No use, distribution or reproduction is permitted which does not comply with these terms. 